FACTA UNIVERSITATIS (NIŠ)

Ser. Math. Inform. Vol. 34, No 2 (2019), 253-260

https://doi.org/10.22190/FUMI1902253K

\title{
TUBULAR SURFACES WITH DARBOUX FRAME IN GALILEAN 3-SPACE
}

\author{
Sezai Kızıltug ${ }^{1}$, Mustafa Dede ${ }^{2}$ and Cumali Ekici ${ }^{3}$
}

(C) 2019 by University of Niš, Serbia | Creative Commons Licence: CC BY-NC-ND

Abstract. In this paper, we define tubular surface by using a Darboux frame instead of a Frenet frame. Subsequently, we compute the Gaussian curvature and the mean curvature of the tubular surface with a Darboux frame. Moreover, we obtain some characterizations for special curves on this tubular surface in a Galilean 3-space.

Keywords. Tubular surface; Darboux frame; Frenet frame; Gaussian curvature.

\section{Introduction}

A Galilean space may be considered as the limit case of a pseudo-Euclidean space in which the isotropic cone degenerates to a plane. This limit transition corresponds to the limit transition from the special theory of relativity to classical mechanics. On the other hand, the Galilean space-time plays an important role in non-relativistic physics. The fact is that the fundamental concepts such as velocity, momentum, kinetic energy, etc., and the principles, laws of motion and conservation laws of classical physics are expressed in terms of the Galilean space[7].

As it is well known, the geometry of space is associated with a mathematical group. The idea of invariance of geometry under transformation groups may imply that on some spacetimes of maximum symmetry there should be a principle of relativity, which requires the invariance of physical laws without gravity under transformations among inertial systems. Surface theory has been a popular topic for many researchers in many aspects $[3,9,8]$. Furthermore, canal surfaces are more popular in computer aided geometric design (CAGD), including designing models of internal and external organs, preparing terrain infrastructures, constructing blending surfaces, reconstructing shape or robotic path planning. Several geometers have studied canal surfaces and tube surfaces and have obtained many interesting results $[4,1,10,2,5]$. Maekawa $[6]$ et al carried out a research on the necessary and

Received April 04, 2017; accepted December 07, 2018

2010 Mathematics Subject Classification. Primary 53A35; Secondary $53 Z 05$ 
sufficient conditions for the regularity of tube surfaces. Besides, Ro and Yoon [10] studied the tubes of the Weingarten type in a Euclidean 3-space. M. Dede [2] studied tube surfaces in a Galilean 3-space. Recently, Dogan and Yayli [4] investigated tubes with a Darboux frame in a Euclidean 3-space. In this study, we investigate tubular surfaces by taking a Darboux frame instead of a Frenet frame in a Galilean 3-space.

\section{Preliminaries}

The Galilean space $G_{3}$ is a Cayley-Klein space equipped with the projective metric of signature $(0,0,+,+)$. The absolute figure of the Galilean space consists of an ordered triple $\{w, f, I\}$, where $w$ is the ideal(absolute) plane, $f$ is the line(absolute line) in $w$ and $I$ is the fixed elliptic involution of points of $f$.

In the non-homogeneous coordinates the similarity group $H_{8}$ has the form

$$
\begin{gathered}
\bar{x} \\
\bar{y}=a_{11}+a_{12} x \\
\bar{z}=a_{21}+a_{22} x+a_{23} y \cos \theta+a_{23} z \sin \theta \\
\bar{z}+a_{32} x-a_{23} y \sin \theta+a_{23} z \cos \theta
\end{gathered}
$$

where $a_{i j}$ and $\theta$ are real numbers[7]. In what follows, the real numbers $a_{12}$ and $a_{23}$ will play the special role. In particular, for $a_{12}=a_{23}=1$, (1) defines the group $B_{6} \subset H_{8}$ of isometries of the Galilean space $G_{3}$.

Planes $x=$ constant are Euclidean and so is the plane $\omega$. Other planes are isotropic. A vector $\mathbf{u}=\left(u_{1}, u_{2}, u_{3}\right)$ is said to be non-isotropic if $u_{1} \neq 0$. All unit non-isotropic vectors are of the form $\mathbf{u}=\left(1, u_{2}, u_{3}\right)$. For isotropic vectors, $u_{1}=0$ holds [7].

Since $x=0$ plane is a Euclidean in Galilean space, it is easy to see that isotropic vectors are on the Euclidean plane.

Definition 1.1. Let $\mathbf{a}=(x, y, z)$ and $\mathbf{b}=\left(x_{1}, y_{1}, z_{1}\right)$ be vectors in a Galilean space. The scalar product is defined as

$$
<\mathbf{a}, \mathbf{b}>=x_{1} x
$$

and the scalar product of two isotropic vectors, $\mathbf{p}=(0, y, z)$ and $\mathbf{q}=\left(0, y_{1}, z_{1}\right)$, is defined as

$$
<\mathbf{p}, \mathbf{q}>_{1}=y y_{1}+z z_{1}
$$

Definition 1.2. Let $\mathbf{u}=\left(u_{1}, u_{2}, u_{3}\right)$ and $\mathbf{v}=\left(v_{1}, v_{2}, v_{3}\right)$ be vectors in a Galilean space [8]. The cross-product of the vectors $\mathbf{u}$ and $\mathbf{v}$ is defined as follows:

$$
\mathbf{u} \wedge \mathbf{v}=\left|\begin{array}{ccc}
0 & e_{2} & e_{3} \\
u_{1} & u_{2} & u_{3} \\
v_{1} & v_{2} & v_{3}
\end{array}\right|=\left(0, u_{3} v_{1}-u_{1} v_{3}, u_{1} v_{2}-u_{2} v_{1}\right)
$$


The curve $\alpha: I \subset \mathbb{R} \rightarrow G_{3}$ of the class $C^{\infty}$ in the Galilean space $G_{3}$ is defined by the parametrization

$$
\alpha(s)=(s, y(s), z(s)),
$$

where $s$ is a Galilean invariant arc-length of $\alpha$. Then the curvature $\kappa(s)$ and the torsion $\tau(s)$ are given by, respectively

$$
\kappa(s)=\sqrt{\ddot{y}(s)^{2}+\ddot{z}(s)^{2}}, \tau(s)=\frac{\operatorname{det}((\dot{\alpha}(s), \ddot{\alpha}(s), \dddot{\alpha}(s))}{\kappa^{2}(s)}
$$

On the other hand, the Frenet vectors of $\alpha(s)$ in $G_{3}$ are defined by

$$
\begin{gathered}
\mathbf{t} \quad=\dot{\alpha}(s)=((1, \dot{y}(s), \dot{z}(s)), \\
\mathbf{n}=\frac{1}{\kappa(s)} \ddot{\alpha}(s)=\frac{1}{\kappa(s)}((0, \ddot{y}(s), \ddot{z}(s)), \\
\mathbf{b} \quad=\frac{1}{\kappa(s)}((0,-\ddot{z}(s), \ddot{y}(s)) .
\end{gathered}
$$

The vectors $\mathbf{t}, \mathbf{n}, \mathbf{b}$ are called the vector of tangent, principal normal and binormal of $\alpha$, respectively. For their derivatives the following Frenet formula is satisfied[8]

$$
\begin{aligned}
\mathbf{t}^{\prime}(s) & =\kappa(s) \mathbf{n}, \\
\mathbf{n}^{\prime}(s) & =\tau(s) \mathbf{b}, \\
\mathbf{b}^{\prime}(s) & =-\tau(s) \mathbf{n} .
\end{aligned}
$$

Since the curve $\alpha(s)$ lies on the surface $M$, there exists another frame along the curve. This new frame is called Darboux frame and is denoted by $\{\mathbf{T}, \mathbf{Y}, \mathbf{N}\}$ where $\mathbf{T}$ is the unit tangent of the curve, $\mathbf{N}$ is the unit normal of the surface $M$ along the curve $\alpha(s)$ and $\mathbf{Y}$ is a unit vector given by $\mathbf{Y}=\mathbf{N} \times \mathbf{T}$. This frame gives us an opportunity to investigate the properties of the curve according to the surface. Since the unit tangent $\mathbf{T}$ is common in both Frenet frame and Darboux frame, the vectors $\mathbf{n}, \mathbf{b}, \mathbf{Y}$ and $\mathbf{N}$ lie on the same plane. The derivative formulae of the Darboux frame of $\alpha(s)$ is given as [9]

$$
\begin{aligned}
& \mathbf{T}^{\prime}(s)=k_{g}(s) \mathbf{Y}+k_{n} \mathbf{N}, \\
& \mathbf{Y}^{\prime}(s)=t_{r}(s) \mathbf{N} \text {, } \\
& \mathbf{N}^{\prime}(s) \quad=t_{r}(s) \mathbf{Y} \text {. }
\end{aligned}
$$

where $k_{g}, k_{n}$ and $t_{r}$ are called the geodesic curvature, the normal curvature and the geodesic torsion, respectively.

Now, we shall mention the surface theory in the Galilean space $G_{3}$.

Let us consider the surface $M$ given by the parametrization

$$
\varphi\left(u^{1}, u^{2}\right)=\left(x\left(u^{1}, u^{2}\right), y\left(u^{1}, u^{2}\right), z\left(u^{1}, u^{2}\right)\right)
$$


where $x\left(u^{1}, u^{2}\right), y\left(u^{1}, u^{2}\right), z\left(u^{1}, u^{2}\right) \in C^{3}$ and $u^{1}, u^{2} \in \mathbb{R}$.

The isotropic unit normal vector field $\mathbf{Z}$ is given by

$$
\mathbf{Z}=\frac{\varphi, 1 \wedge \varphi, 2}{\left\|\varphi, 1 \wedge \varphi_{, 2}\right\|_{1}}
$$

where $w=\left\|\varphi_{, 1} \wedge \varphi_{, 2}\right\|_{1}$ and the partial differentiation with respect to $u^{2.1}$ and $u^{2.2}$ denoted by suffixes 1 and 2 , respectively.

The first fundamental form of the surface is defined as

$$
I=\left(g_{i j}+\varepsilon h_{i j}\right) d u^{i} d u^{j}
$$

where

$$
g_{i j}=\left\langle\varphi_{, i}, \varphi_{, j}\right\rangle, h_{i j}=\left\langle\varphi_{, i}, \varphi_{, j}\right\rangle_{1}
$$

and $\varepsilon$ is

$$
\epsilon=\left\{\begin{array}{lll}
0, & d v^{1}: d v^{2} & \text { non-isotropic } \\
1, & d v^{1}: d v^{2} & \text { isotropic }
\end{array}\right.
$$

The coefficients $L_{i j}$ of the second fundamental form are given by

$$
L_{i j}=<\frac{\varphi_{, i j} x_{, 1}-x_{, i j} \varphi_{, 1}}{x_{, 1}}, \mathbf{Z}>_{1}
$$

Finally, the Gauss curvature $K$ and the mean curvature $H$ of the surface $M$ are defined as

$$
\begin{gathered}
K \quad=\frac{\operatorname{det} L_{i j}}{w^{2}}=\frac{L_{11} L_{22}-L_{12}}{w^{2}} \\
2 H \quad=g^{i j} L_{i j}=g^{11} L_{11}+g^{12} L_{12}+g^{22} L_{22}
\end{gathered}
$$

respectively, where

$$
g^{11}=\frac{g_{22}}{w^{2}}, g^{12}=-\frac{g_{12}}{w^{2}}, g^{22}=-\frac{g_{11}}{w^{2}}
$$

\section{Tubular surface with Darboux frame in $G_{3}$}

M.Dede defined the tubular surface by using a Frenet frame in a Galilean 3space. In this section, we define tubular surface by using a Darboux frame instead of a Frenet frame.

Let the center curve $\alpha(s)$ be on the surface $M$. The characteristic circles of the canal surface lie in the plane which is perpendicular to the tangent of the center curve $\alpha(s)$. In light of this definition, the tubular surface can be defined by using a Darboux frame as

$$
M(s, \beta)=\alpha(s)+r(\cos \beta \mathbf{Y}+\sin \beta \mathbf{N})
$$


Using (2.6) we get partial derivatives of $M$ with respect to $s$ and $\beta$ as follows:

$$
\begin{gathered}
M_{s}=T+r\left(\cos \beta\left(t_{r} \mathbf{N}\right)-\sin \beta\left(t_{r} Y\right)\right) \\
M_{\beta} \quad=-r \sin \beta \mathbf{Y}+r \cos \beta \mathbf{N}
\end{gathered}
$$

The cross-product of these two vectors is given as

$$
M_{s} \wedge M_{\beta}=-r \cos \beta \mathbf{Y}-r \sin \beta \mathbf{N}
$$

Using (2.8) and (3.5), we obtain an isotropic normal vector of the tubular surface as

$$
\mathbf{Z}=-\cos \beta \mathbf{Y}-\sin \beta \mathbf{N}
$$

From (2.13) and (3.5), we obtain the first fundamental form of the tubular surface with a Darboux frame in a Galilean space as

$$
I=d s^{2}+\epsilon r^{2} d \beta^{2}
$$

where $\epsilon$ is

$$
\epsilon= \begin{cases}0, & d u \neq 0 \\ 1, & d u=0\end{cases}
$$

The second order partial differentials of the surface $M$ are obtained as

(3.8) $M_{s s}=\left(k_{g}-r \cos \beta t_{r}^{2}-r \sin \beta t_{r}^{\prime}\right) \mathbf{Y}+\left(k_{n}+r \cos \beta t_{r}^{\prime}-r \sin \beta t_{r}^{2}\right) \mathbf{N}$,

(3.9) $M_{\beta s}=\left(-r \cos \beta t_{r}\right) \mathbf{Y}+\left(-r \sin \beta t_{r}\right) \mathbf{N}$,

$$
\text { (3.10) } M_{\beta \beta} \quad=-r \cos \beta \mathbf{Y}-r \sin \beta \mathbf{N}
$$

Equations (3.12), (3.13) and (3.1) lead to the coefficients of the second fundamental form obtained by,

$$
\begin{gathered}
L_{11}=-\cos \beta k_{g}-k_{n} \sin \beta+r t_{r}^{2}, \\
=r t_{r}, \\
L_{12} \\
L_{22} \\
=r .
\end{gathered}
$$

Thus, the Gaussian curvature $K$ is given by:

$$
K=\frac{-\cos \beta k_{g}-k_{n} \sin \beta}{r}
$$

From the equations (2.11), (3.4) and (3.5), we get

$$
g^{11}=g^{12}=0, g^{22}=\frac{1}{r^{2}}
$$

Then, substituting (3.14), (3.15) and (3.16) into (3.1), we obtain the mean curvature of the tubular surface as

$$
H=\frac{1}{2 r}
$$




\section{Some Characterizations for Special Curves on Tubular Surfaces in $G_{3}$}

In this section, we investigate the relation between parameter curves and special curves such as geodesic curves, asymptotic curves, and lines of curvature on this tube surface $M(s, \beta)$ in a Galilean space

Theorem 3.1 Let $M(s, \beta)$ be a tubular surface in $G_{3}$. Then

i) $\beta$ - parameter curves are also geodesic.

ii) $s$ - parameter curves are also geodesic if and only if $k_{g}, k_{n}$ and $t_{r}$ of $\alpha(s)$ satisfy the equation:

$$
-\cos \beta k_{n}+\sin \beta k_{g}-r t_{r}^{\prime}=0
$$

\section{Proof:}

i) For $s-$ and $\beta$ - parameter curves, we get

$$
\begin{gathered}
\mathbf{Z} \wedge M_{\beta \beta}=(-\cos \beta \mathbf{Y}-\sin \beta \mathbf{N}) \wedge(-r \cos \beta \mathbf{Y}-r \sin \beta \mathbf{N}) \\
=r \cos \beta \sin \beta T-r \sin \beta \cos \beta T=0 .
\end{gathered}
$$

Since $Z \wedge M_{\beta \beta}=0$, it follows that $\beta$ - parameter curves are geodesic.

ii)

$$
\mathbf{Z} \wedge M_{s s}=\left(-\cos \beta k_{n}+\sin \beta k_{g}-r t_{r}^{\prime}\right) \mathbf{T}(s)
$$

It is easy to see that $\mathbf{Z} \wedge M_{s s}=0$ if and only if $-\cos \beta k_{n}+\sin \beta k_{g}-r t_{r}^{\prime}=0$. This completes the proof.

Corollary 3.1 Let $\alpha(s)$ be a geodesic curve on the tubular surface $M(s, \beta)$ in $G_{3}$. If $s$ - parameter curves are also geodesic on $M(s, \beta)$, then the curvatures $\kappa$ and $\tau$ of $\alpha(s)$ satisfy the equation:

$$
\cos \beta \kappa-r \tau^{\prime}=0
$$

Proof: Since the center curve $\alpha(s)$ is geodesic curve, we have $k_{g}=0, k_{n}=\kappa$ and $t_{r}=\tau$. Substituting $k_{g}=0, k_{n}=\kappa$ and $t_{r}=\tau$ in (4.1) the equation, we get

$$
\cos \beta \kappa-r \tau^{\prime}=0
$$

Hence, the proof is completed.

Corollary 3.2 Let $\alpha(s)$ be an asymptotic curve on the tubular surface $M(s, \beta)$ in $G_{3}$. If $s$ - parameter curves are also geodesic on $M(s, \beta)$, then the curvatures $\kappa$ and $\tau$ of $\alpha(s)$ satisfy the equation:

$$
\sin \beta \kappa-r \tau^{\prime}=0
$$


Proof: Since the center curve $\alpha(s)$ is an asymptotic curve, we have $k_{n}=0$, $k_{g}=\kappa$ and $t_{r}=\tau$. Substituting $k_{n}=0, k_{g}=\kappa$ and $t_{r}=\tau$ in (4.1), we get

$$
\cos \beta \kappa-r \tau^{\prime}=0
$$

Hence, the proof is completed.

Theorem 3.2 For the tubular surface $M(s, \beta)$ in $G_{3}$.

i) $\beta$ - parameter curves cannot be asymptotic curves.

ii) $s$ - parameter curves are also geodesic if and only if $M(s, \beta)$ is generated by a moving sphere with the radius function

$$
r=\frac{\cos \beta k_{g}+\sin \beta k_{n}}{t_{r}^{\prime}} .
$$

Proof: i) Since $\left\langle\mathbf{Z}, M_{\beta \beta}\right\rangle=r \neq 0, \beta$ - parameter curves cannot be asymptotic curves on $M(s, \beta)$

ii) $s$-parameter curves are also asymptotic curve on $M(s, \beta)$ if and only if

$$
\left\langle\mathbf{Z}, M_{s s}\right\rangle=-\cos \beta k_{g}-\sin \beta k_{n}+r t_{r}^{\prime}=0 .
$$

Thus, we get the radius function:

$$
r=\frac{\cos \beta k_{g}+\sin \beta k_{n}}{t_{r}^{\prime}} .
$$

This completes the proof.

Corollary 3.3 Let $s$ - parameter curves are also asymptotic curves on $M(s, \beta)$ in $G_{3}$.

i) If the center curve $\alpha(s)$ is a geodesic curve on $M(s, \beta)$, then

$$
r=\frac{\sin \beta \kappa}{\tau^{\prime}}
$$

ii) If the center curve $\alpha(s)$ is an asymptotic curve on $M(s, \beta)$, then

$$
r=\frac{\cos \beta \kappa}{\tau^{\prime}}
$$

\section{R E F E R E N C E S}

1. H. S. Abdel-Aziz and M. Khalifa SaAd: Weingarten Timelike Tube Surfaces Around a Spacelike Curve, Int. J. Math. Anal., 5, (2011), 1225-1236.

2. M. DEDE: Tubular surfaces in Galilean space, Math. Commun., 18, 2013, 209217. 
3. B. Divjak and Z. MiLin: Sipus Minding isometries of ruled surfaces in pseudoGalilean Space, J. Geom., 77, (2003), 35-47.

4. F. DoĞAN and Y. YAYLI: Tubes with Darboux Frame, Int. J. Contemp.Math. Sci., 7(16), (2012), 751-758.

5. S. KiziltuĞ and Y. YAYli: Timelike tubes with Darboux frame in Minkowski3space, International Journal of Physical Sciences, 8(1), (2013), 31-36.

6. T. Maekawa, M. N. Patrikalakis, T. Sakkalis and G. Yu: Analysis and Applications of Pipe Surfaces, Comput. Aided Geom. Des., 15 (1998), 437-458.

7. J. S. Ro and D. W. Yoon: Tubes of Weingarten types in a Euclidean 3-space, Journal of the Chungcheong Mathematical Society, 22(3), (2009), 359-366.

8. Z. M. Sipus: Ruled Weingarten surfaces in Galilean Space, Period. Math. Hung., 56, (2008), 213-225.

9. T. ŞAHIN: Intrinsic equations for a generalized relaxed elastic line on an oriented surface in the Galilean space, Acta Mathematica Scientia, 33B(3), (2013), 701711.

10. I. M. Yaglom: A Simple Non-Euclidean Geometry and Its Physical Basis, Springer, New York, USA, 1979.

Sezai Kızıltug

Faculty of Science

Department of Mathematics

Erzincan Binali Yıldırım University

24000 Erzincan, Turkey

skiziltug@erzincan.edu.tr

Mustafa Dede

Faculty of Science

Department of Mathematics

Kilis 7 Aralık University

79000 Kilis, Turkey

mustafadede@kilis.edu.tr

Cumali Ekici

Faculty of Science

Department of Mathematics-Computer

Eskişehir Osmangazi University

26480 Eskişehir, Turkey

cekici@ogu.edu.tr 